\title{
Application of FURIA for Finding the Faults in a Hydraulic Brake System Using a Vibration Analysis through a Machine Learning Approach
}

\author{
Alamelu Manghai T. $\mathrm{M}^{1}$, and Jegadeeshwaran $\mathrm{R}^{2}$ \\ ${ }^{1,2}$ School of Mechanical and Building Science, Vellore Institute of Technology, Chennai-600127, India. \\ lalamelumangai.m@vit.ac.in \\ ${ }^{2}$ jegadeeshwaran.r@vit.ac.in
}

\begin{abstract}
Vibration-based continuous monitoring system for fault diagnosis of automobile hydraulic brake system is presented in this study. This study uses a machine learning approach for the fault diagnosis study. A hydraulic brake system test rig was fabricated. The vibration signals were acquired from the brake system under different simulated fault conditions using a piezoelectric transducer. The histogram features were extracted from the acquired vibration signals. The feature selection process was carried out using a decision tree. The selected features were classified using fuzzy unordered rule induction algorithm (FURIA) and Repeated Incremental Pruning to Produce Error Reduction (RIPPER) algorithm. The classification results of both algorithms for fault diagnosis of a hydraulic brake system were presented. Compared to RIPPER and J48 decision tree, the FURIA performs better and produced $98.73 \%$ as the classification accuracy.
\end{abstract}

Keywords: FURIA, RIPPER, histogram features, J48 decision tree algorithm, confusion matrix.

\section{INTRODUCTION}

A brake is an important controlling element which consists of a combination of interacting parts that work to slow down a vehicle. Any failure in the brake system makes an impact on vehicle stability and the passenger's safety. Hence, preventive maintenance of the hydraulic brake system is crucial in order to avoid damage. This is achieved through the condition monitoring process. Condition monitoring is defined as the continuous evaluation of the health of the part and equipment throughout its service life. It is possible to

\footnotetext{
Alamelu Manghai et al. This is an open-access article distributed under the terms of the Creative Commons Attribution 3.0 United States License, which permits unrestricted use, distribution, and reproduction in any medium, provided the original author and source are credited.
}

provide adequate warning of inevitable failure in the brake system through condition monitoring (Jegadeeshwaran \& Sugumaran, 2015). Furthermore, it is likewise possible to schedule a future preventive maintenance. Using vibration analysis, the condition of a machine can be constantly monitored (Shon \& Charles, 2011). The health of a machine can be analyzed through a detailed study. This may be used to identify any faults that may be arising or that already exist. Machine learning is one of the most efficient condition monitoring approaches which can be used for such analysis in order to predict the faults well in advance (Ravikumar, Ramachandran \& Sugumaran, 2011). Generally, vibration signal (Ragini, Prakash \& Gopal 2015) and acoustic emission (Faris et al., 2015) are the two major elements which contain features as information. There are many features like statistical (Jegadeeshwaran \& Sugumaran, 2013), histogram (Chapelle et al., 1999), wavelets (Livani \& Yaman, 2011), AR-MA (Erdem \& Jing, 2011), etc. The machinery condition is directly related to the vibration generated. In many fault diagnosis study, the vibration signals have been successfully used for monitoring the condition of rotating machine elements like bearings (Jafar, Mohammad \& Hamid, 2014), pumps (Sakthivel, Sugumaran \& Babudevasenapati, 2010), brakes (Jegadeeshwaran \& Sugumaram, 2015), etc. Hence, the vibration signal under various fault conditions has been considered for the learning process in this study.

From the acquired vibration signal, the histogram features were extracted to make the fault diagnosis study. After feature extraction, the feature selection was carried out. Many techniques have been reported for feature selection. Among this decision tree generated from the J48 decision tree algorithm is the most generally suggested technique for the feature selection process (Sugumaran, Muralidharan \& Ramachandran, 2007). In this study, the decision tree and the effect of a number of features study were used for feature selection. 
The feature classification is the final stage of machine learning. The learning of rule-based classification models has been a functioning area of research for a long time. Many learning algorithms such as Logit Boost (Brake fault diagnosis) (Alamelu, Jegadeeshwaran \& Sugumaran, 2018), decision tree (Gearbox fault diagnosis) (Saravanan, Cholairajan, \& Ramachandran, 2009), best first tree (Brake fault diagnosis) (Jegadeeshwaran \& Sugumaran, 2013), Bayes and Naïve Bayes (Pump fault diagnosis) (Sugumaran, Muralidharan, \& Ramachandran, 2007), K Star (Brake fault diagnosis and tool condition monitoring) (Painuli, Elangovan \& Sugumaran, 2012) have been reported in literature for the fault diagnosis study. The greater part of the learning model delivers the decision list dependent on which the decision is being made. The rules are gained from the smallest one to the second biggest to create such decision list. A new query is classified using the primary rules that are produced. In literature, one such govern based fuzzy model was recommended for the brake fault diagnosis study (Jegadeeshwaran \& Sugumaran 2015). Since there are many rules, the sorting of rules based on the priority is a challenging one. Hence a novel Fuzzy unordered Rule Induction Algorithm (FURIA) approach has been proposed for the classification. In FURIA, an unordered rule set is used for each class (Ana Palacios et al., 2016). The computational effort during the learning stage in FURIA is very less compared to other approaches. Hence, in the present study, the FURIA model was used for the classification and the results were compared with the RIPPER and J48 decision tree.

The paper has been structured as follows:

In section 2, the experimental setup and experimental procedure have been reported for acquiring the vibration signal under both good as well as faulty conditions. In section 3, the feature extraction process has been reported. The feature selection process has been explained in section 4. The basics of FURIA and RIPPER algorithms have been recalled in Section 5. Section 6 is devoted to the results and discussion. The selected features were classified using Fuzzy, Ripper and FURIA algorithms. It is shown that FURIA significantly outperforms the original RIPPER, as well as a fuzzy classifier in terms of classification accuracy.

\section{EXPERIMENTAL STUDIES}

The effectiveness of the proposed work has been investigated through an experimental study. In this experimental study, the health condition of the brake system was diagnosed using the histogram features under the various fault conditions. These studies were discussed in the following subsections.

- Experimental Setup

- Experimental Procedure

\subsection{Experimental Setup}

A commercial vehicle's hydraulic brake system was fabricated as shown in Fig 1. The drive shaft which is driven by a variable speed DC motor consists of both disc and drum brake on the same shaft. The piezoelectric type accelerometer of $500 \mathrm{~g}$ range, $10 \mathrm{mV} / \mathrm{g}$ sensitivity and 40 $\mathrm{kHz}$ resonant frequency was used as a transducer for obtaining vibration signals. The vibration signal was acquired through a data acquisition device (NI 9234, 4 Channel, 51.2 kilo Samples /sec) through NI-LabVIEW.

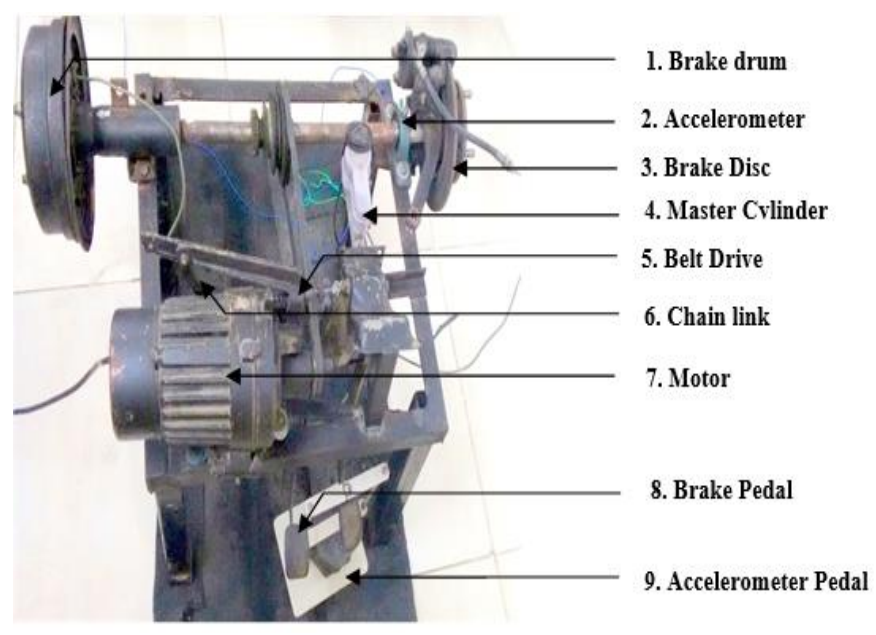

Fig. 1. Experimental setup - Brake fault diagnosis

\subsection{Experimental procedure}

Initially, all the brake components were in good condition. Under these circumstances, the vibration signals were acquired by using an accelerometer with the following specifications.

1. Sample length $-10,000$ (Arbitrarily chosen)

2. Sampling frequency $-24 \mathrm{kHz}$ (As per Nyquist sampling theorem).

3. Number of samples - Minimum of 55 trails

4. Wheel speed $-667 \mathrm{rpm}$ (constant speed)

The following nine fault conditions were simulated on the brake system (Jegadeeshwaran \& Sugumaran 2013).

1. Brake Oil Spill (BOS)

2. Air bubbles in the brake fluid (AIR)

3. Pad Wear on Drum Brake (DRPW)

4. Reservoir Leak (RL)

5. Even Pad Wear on Disc Brake- Inner (DPWI)

6. Uneven Pad Wear on Disc Brake (UDPWI) - Inner

7. Even Pad Wear on Disc Brake - Inner and Outer (DPWIO)

8. Uneven Pad Wear on Disc Brake (UDPWI) - Inner

9. Mechanical Fade on the Drum Brake 
Fig 2 (a) shows the drum brake pad wear condition, Fig 2 (b) shows the disc brake pad wear condition and Fig 2 (c) shows the drum brake mechanical fade condition. Fig. 3 shows the experimental procedure for acquiring the vibration signals.

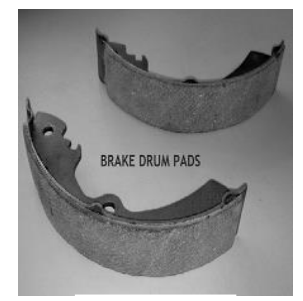

2 (a)

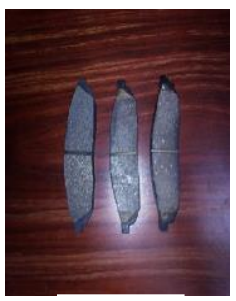

2(b)

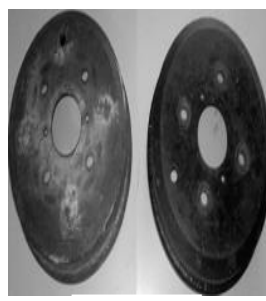

2(c)
Fig. 2(a). Drum brake pads wear condition, (b) Disc brake pads wear condition, (c) Drum brake mechanical fade

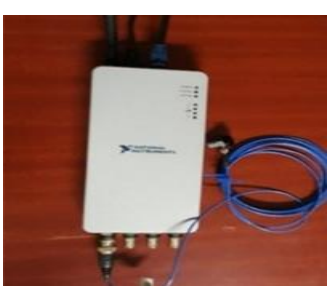

DAQ Hardware

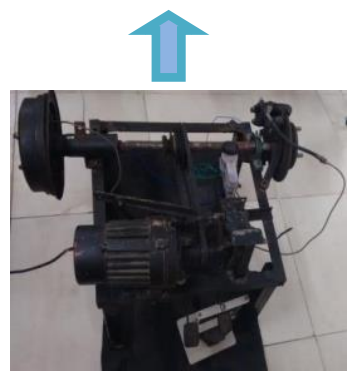

Brake Setup

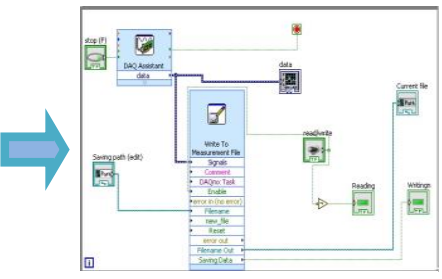

LabVIEW Program

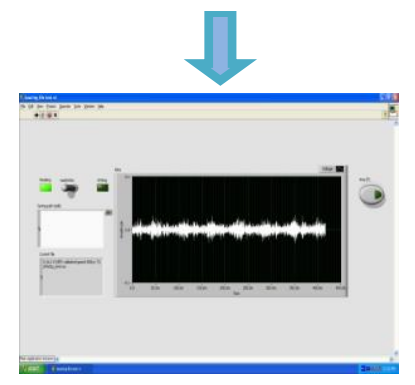

Acquired vibration signal
Fig. 3. Experimental Procedure

\section{Feature EXTRACTION}

Extracting specific information from the raw vibration signals are called feature extraction (Sakthivel et al., 2011). Histogram feature extraction process was carried out in this study. The histogram is a representation of the numerical data distribution (Pearson, 1985). It is a probability distribution of a continuous variable. The bin width and bin range were used for plotting the histogram. The bin (range of values) is selected from the statistical features such as maximum (7.830098) and minimum (-6.831523).

The value between the maximum and minimum of the vibration signal was divided by a number of frequency ranges. The range between the maximum and minimum value of the signal is divided as 69 bins ( 2 to 70 bin range).
Figure 4 shows the sample histogram extracted from the acquired vibration signal for 7 bin range under good condition of the brake system. In this study, a histogram features were extracted using the visual basic code as shown in Figure. 5.

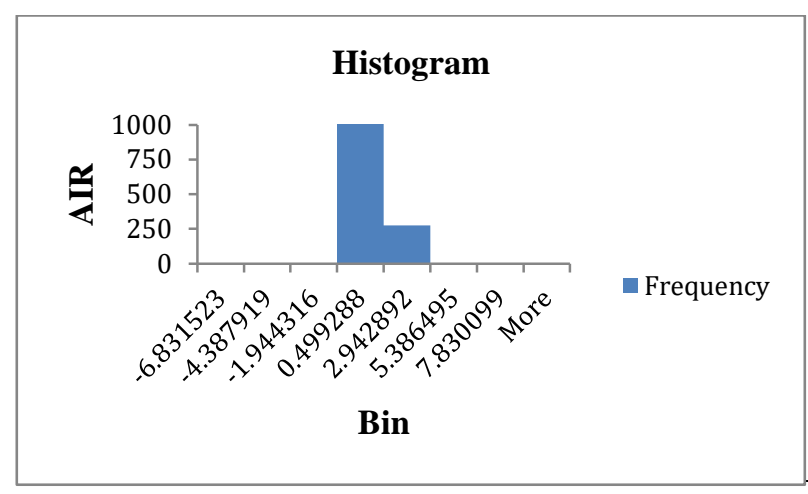

Fig. 4. Histogram of vibration signal with air in the reservoir condition

The sample VB program used for extracting the histogram for each bin range is given below:

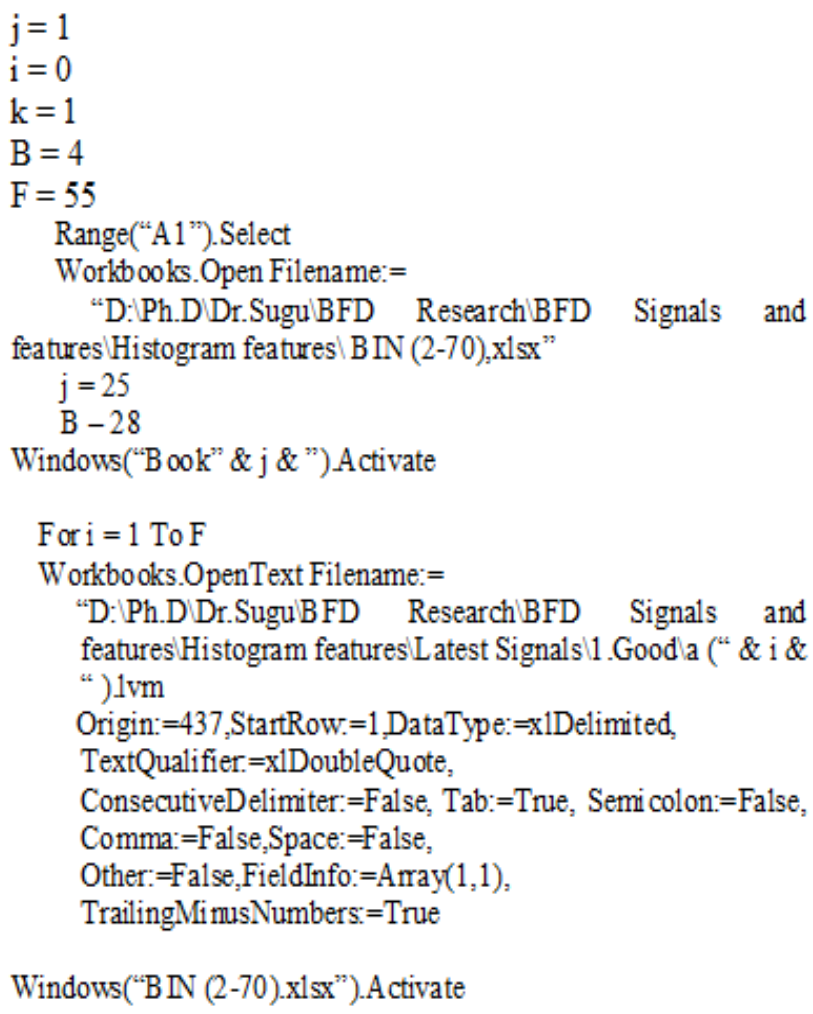

Fig. 5. Histogram Features extraction using Visual Basic

\section{Feature Selection}

The decision tree was used for selecting the features from extracted features. For classification, only the selected 
features are contributing and other do not. All the extracted 69 bin ranges (2-70) were classified one by one using a J48 decision tree algorithm. Fig. 6 shows the classification accuracy for various bin ranges.

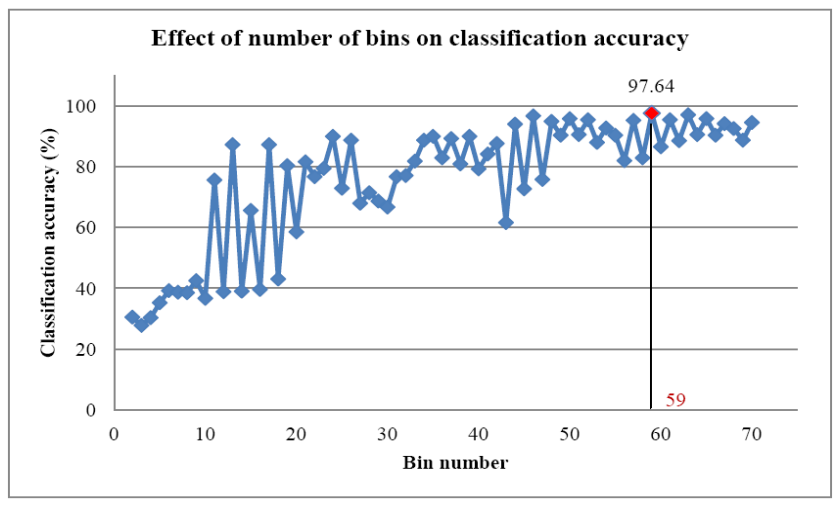

Fig. 6. Effect of a number of bins on classification accuracy.

Referring Fig. 6, the classifier gives maximum classification accuracy for the $59^{\text {th }}$ bin (frequency) ranges. For the $59^{\text {th }}$ bin, the decision tree was generated as shown in Fig. 7. The features which dominate represent the brake condition descriptors. The level of contribution by individual features is given by a statistical measure within the parenthesis in the decision tree (Fig. 7).

From the decision tree, only seven contributors were identified as dominant amongst the available features. The contributing bin values are namely, H22, H24, H27, H28, $\mathrm{H} 29, \mathrm{H} 30$ and H33. However, the feature H22 has less contribution to the classification compared to the others. As the feature $\mathrm{H} 22$ has no effect on classification accuracy, it was eliminated. Finally, the top six features, namely, H24, $\mathrm{H} 27, \mathrm{H} 28, \mathrm{H} 29, \mathrm{H} 30$, and $\mathrm{H} 33$ have been selected for classification. The J48 decision tree produced a better classification accuracy as $97.64 \%$ for the selected six bin ranges. The weka - a datamining machine learning software was used for the feature selection and classification.

\section{Feature Classification}

In the present study, the classifier that maps the set of extracted features to the condition of the brake system was considered for the fault diagnosis study. The RIPPER and FURIA have been used as a classifier model.

\subsection{Repeated Incremental Pruning to Produce Error Reduction (RIPPER) Algorithm}

RIPPER is an optimized version of IREP. In RIPPER, an initial rule set is generated and simplified using the pruning operators. Typical pruning operators are used for reducing the error on the pruning set. Simplification ends at the moment when the error rate is increased. The Ripper algorithm may be implemented in two stages:

1) Building stage: In this stage, the rule set is generated until it reaches the description length (DL) of the rule set mentioned. This is done in two ways

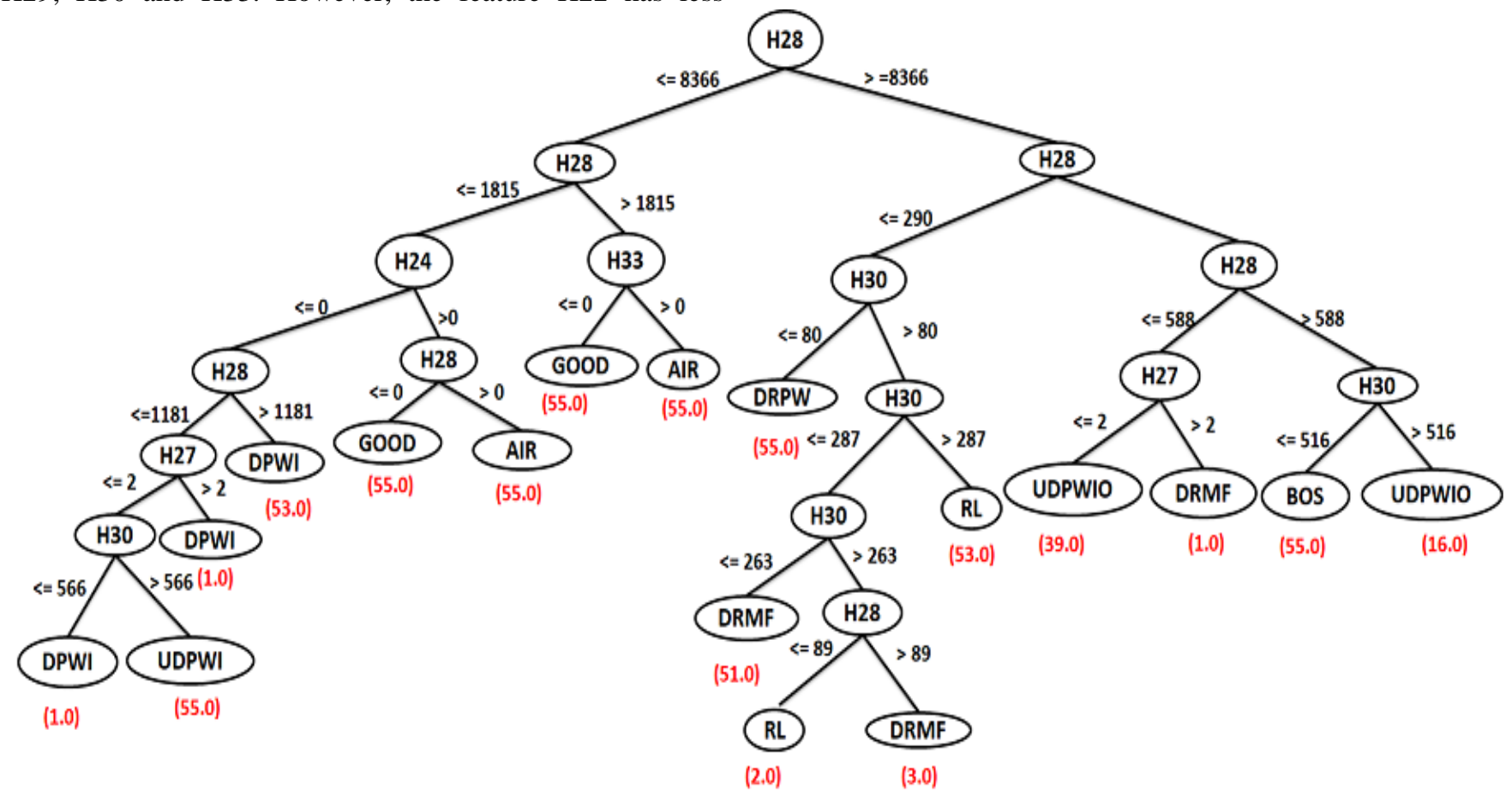

Fig. 7. Decision tree with histogram features 
(i) Growing phase: The rules are grown by adding the conditions until transforming into perfect result

(ii) Pruning Phase: The generated rule is simplified using pruning so as to maximize its performance on the pruning data.

(iii) Optimization stage: In this stage, the minimum description length criterion is used to examine the rules. More rules are generated based on the residual positives using Building Stage again. The algorithm iterates ' $k$ ' times for optimizing the rule set (Pan et al., 2018). Fig 8 shows the RIPPER algorithm flowchart

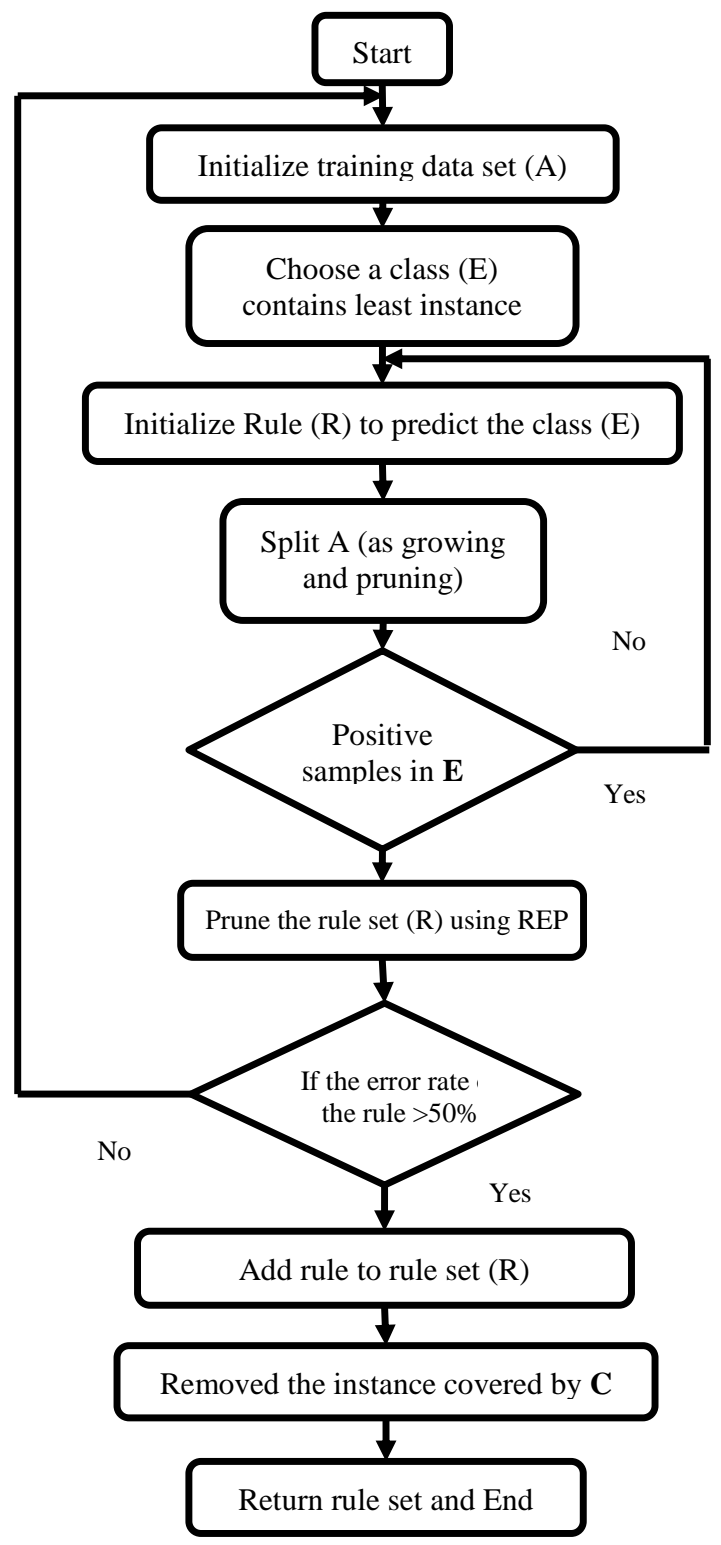

Fig 8: RIPPER algorithm flowchart

\subsection{Fuzzy Unordered Rule Induction Algorithm (FURIA)}

FURIA is a modified version of the RIPPER algorithm. In the RIPPER algorithm, a default rule is used for learning. But FURIA proposes a rule set to learn for each class (Bostrom, 2004). The initial rule set is learned on the whole training data directly with the unpruned rule set. Hence, there is no pruning phase in FURIA. However, the pruning phase is retained in the optimization stage to minimize the MDL. The removal of pruning doesn't affect the classification accuracy.

\subsubsection{Rule Fuzzification}

The rules obtained from the modified RIPPER algorithm is fuzzified to get the fuzzy rules. The rules are fuzzified until repeated all antecedents have been covered. Flowchart of FURIA algorithm is shown in Fig. 9.

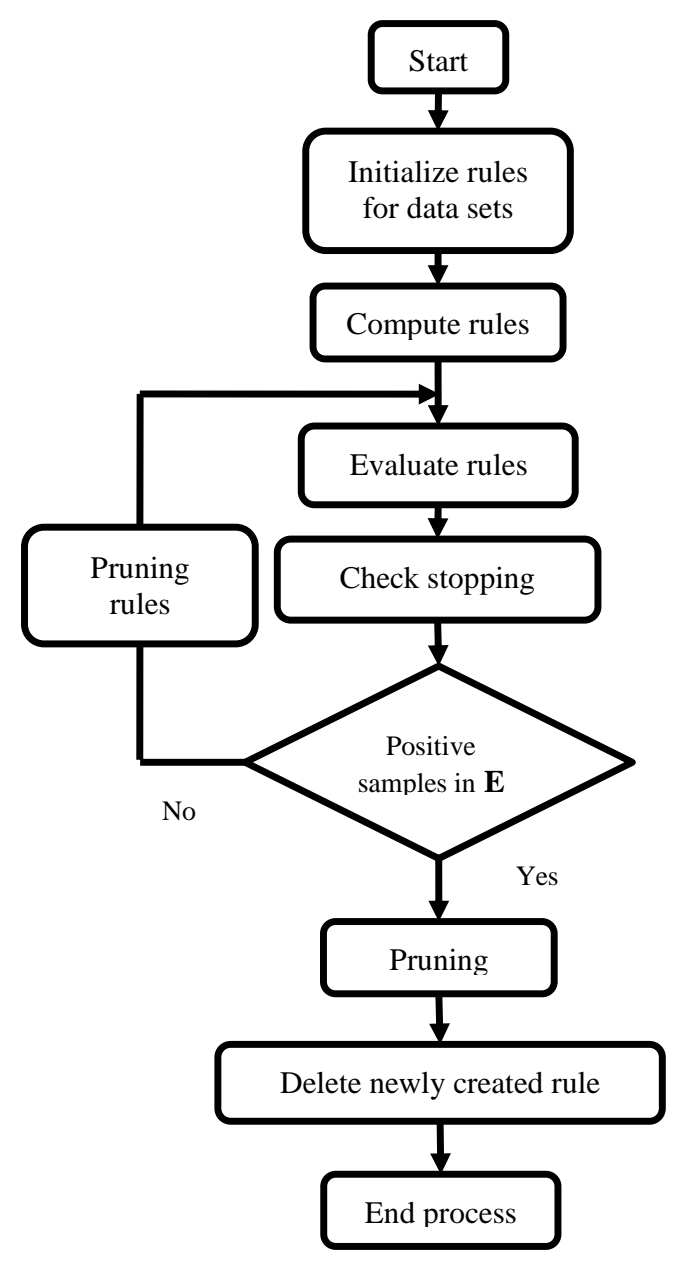

Fig 9: FURIA algorithm flowchart 


\subsubsection{Classification and Rule Stretching}

For a new query, the fuzzy rules have been learned for each class using a certainty factor. In the case, the query is not covered by any rule, and then the classification is done using the rule stretching method. To address this non covering problem, all the fuzzy rules are replaced by their minimal generalizations. After deriving all minimal generalizations, each rule was re-evaluated using Laplace accuracy on the training data and then the query was classified using the rule with the highest evaluation. However, this rule stretching process requires huge computational complexity. Eineborg and Bostrom proposed a new methodology to overcome these demerits. In the proposed approach, the pruned rules which reduce the relevance of the rule for the query has been removed. The remaining conditions are corrected using Laplace theory. Hence, longer and more specific rules were derived. Computational effort to this rule stretching is minimal.

\section{RESUlts AND Discussion}

From the experimental setup the vibration signals were acquired for various conditions. Histogram feature extraction techniques were used for extracting the vibration signal under each condition. From this the contributing features were selected using the decision tree algorithm. The selected features were classified using the RIPPER and FURIA algorithm as discussed below feature selection and feature classification process have been explained in this study.

\subsection{Feature Classification using RIPPER algorithm}

From the experimental setup, the raw vibration signal was extracted for the various fault conditions. From the vibration signal 69 bin ranges were extracted. Among the 69 bins, $59^{\text {th }}$ bin produces maximum classification accuracy. Hence Bin 59 was chosen for finding the classification accuracy. From the $59^{\text {th }}$ bin, only seven ranges were chosen as contributing features. The selected seven features were classified using the RIPPER algorithm. The RIPPER algorithm generates 13 rules based on which the classification accuracy was calculated. The following parameters were used for classification:

$\begin{array}{lll}\text { Batch Size } & : & 100 \\ \text { Folds } & : & 3 \\ \text { MinNo. } & : & 3.0 \\ \text { Optimizations } & : & 30 \\ \text { Seed } & : & 1\end{array}$

JRIP rules:

1. $(\mathrm{H} 30>=2284)$ and $(\mathrm{H} 27<=4) \Rightarrow$ Condition=GOOD $(55.0 / 0.0)$

2. $(\mathrm{H} 29>=9608)$ and $(\mathrm{H} 30>=116)$ and $(\mathrm{H} 30<=$ 262) $=>$ Condition $=$ DRMF $(49.0 / 0.0)$
1. $(\mathrm{H} 29>=9523)$ and $(\mathrm{H} 28>=111) \Rightarrow$ Condition=DRMF (5.0/0.0)

2. $(\mathrm{H} 28>=1203)$ and $(\mathrm{H} 27<=68) \Rightarrow$ Condition=DPWI (53.0/0.0)

3. $(\mathrm{H} 29>=8458)$ and $(\mathrm{H} 28>=326)$ and $(\mathrm{H} 28<=$ $580)=>$ Condition=UDPWIO (40.0/1.0)

4. $(\mathrm{H} 30>=537)$ and $(\mathrm{H} 29>=8458)$ and $(\mathrm{H} 28>=$ $617)=>$ Condition=UDPWIO $(16.0 / 0.0)$

5. $(\mathrm{H} 29<=1235) \Rightarrow$ Condition=DPWIO $(55.0 / 0.0)$

6. $(\mathrm{H} 30<=504)$ and $(\mathrm{H} 28>=598) \Rightarrow$ Condition=BOS $(55.0 / 0.0)$

7. $(\mathrm{H} 27>=220)=>$ Condition=AIR $(55.0 / 0.0)$

8. $(\mathrm{H} 28>=935)=>$ Condition=UDPWI $(57.0 / 2.0)$

9. $(\mathrm{H} 30<=80)=>$ Condition=DRPW $(55.0 / 0.0)$

10. $(\mathrm{H} 30>80)=>$ Condition $=\mathrm{RL}(55.0 / 0.0)$

Number of Rules: 12

The 12 rules were used for the classification process and the classification accuracy was found. The classification accuracy is generally described in the form of confusion matrix as shown in Table 1.

\begin{tabular}{|c|c|c|c|c|c|c|c|c|c|c|}
\hline Category & $\boldsymbol{A}$ & $\boldsymbol{B}$ & $\boldsymbol{C}$ & $\boldsymbol{D}$ & $\boldsymbol{E}$ & $\boldsymbol{F}$ & $\boldsymbol{G}$ & $\boldsymbol{H}$ & $\boldsymbol{I}$ & $\boldsymbol{J}$ \\
\hline $\boldsymbol{A}$ & 54 & 0 & 0 & 0 & 1 & 0 & 0 & 0 & 0 & 0 \\
\hline $\boldsymbol{B}$ & 0 & 55 & 0 & 0 & 0 & 0 & 0 & 0 & 0 & 0 \\
\hline $\boldsymbol{C}$ & 0 & 0 & 53 & 0 & 0 & 1 & 1 & 0 & 0 & 0 \\
\hline $\boldsymbol{D}$ & 0 & 1 & 0 & 51 & 1 & 1 & 1 & 0 & 0 & 0 \\
\hline $\boldsymbol{E}$ & 0 & 0 & 1 & 0 & 54 & 0 & 0 & 0 & 0 & 0 \\
\hline $\boldsymbol{F}$ & 0 & 0 & 0 & 0 & 1 & 54 & 0 & 0 & 0 & 0 \\
\hline $\boldsymbol{G}$ & 0 & 0 & 0 & 0 & 1 & 1 & 52 & 1 & 0 & 0 \\
\hline $\boldsymbol{H}$ & 0 & 0 & 0 & 0 & 0 & 0 & 0 & 54 & 0 & 1 \\
\hline $\boldsymbol{I}$ & 0 & 0 & 0 & 0 & 0 & 0 & 2 & 0 & 52 & 1 \\
\hline $\boldsymbol{I}$ & 0 & 0 & 0 & 0 & 0 & 0 & 1 & 0 & 2 & 52 \\
\hline A: GOOD; $:$ AIR; C: BOS; D: DPWI; E: DBPIO; F: UDPWI; $:$ \\
\hline
\end{tabular}

Table 1. Confusion matrix for the RIPPER algorithm

The diagonal elements in the confusion matrix show the correctly classified attributes. The first row in the confusion matrix belongs to the "GOOD" condition data points. The first element in the first row belongs to GOOD condition. In this 54 data points have been correctly classified. Other than diagonal elements show the misclassification details. There are one misclassified data in the $5^{\text {th }}$ column which belongs to disc brake pad wear Uneven - Inner (UDPWI) condition. The second element in the second row belongs to "AIR (Air in reservoir)" condition. In this case all the 55 data points are correctly classified. Other than the diagonal elements is zero. Hence there is no misclassification. Likewise the misclassification can be clearly identified using the confusion matrix. Totally 550 data points were considered for all the 10 conditions. Among them 531 were correctly classified and 19 were misclassified. The summary of the classification has been given below. 
Classification summary

Total Number of data point

Correctly classified

Misclassified

KS

MA error

RMS error

RA error

KS: Kappa Statistic; MA: Mean Absolute; RMS: Root mean square; RA: Relative absolute

Table 2 shows the detailed accuracy by the class. The classification and misclassification details can be studied by referring to the table. True positive rate, precision, recall, ROC area, and PRC area should be one for an ideal classifier. The effectiveness of the classifier model with individual features can be studied using this table.

\begin{tabular}{|c|c|c|c|c|c|c|c|c|}
\hline $\boldsymbol{A}$ & $\boldsymbol{B}$ & $\boldsymbol{C}$ & $\boldsymbol{D}$ & $\boldsymbol{E}$ & $\boldsymbol{F}$ & $\boldsymbol{G}$ & $\boldsymbol{H}$ & Class \\
\hline 0.982 & 0 & 1 & 0.982 & 0.991 & 0.99 & 0.991 & 0.984 & $\boldsymbol{A}$ \\
\hline 1 & 0.002 & 0.982 & 1 & 0.991 & 0.99 & 0.999 & 0.981 & $\boldsymbol{B}$ \\
\hline 0.964 & 0.002 & 0.981 & 0.964 & 0.972 & 0.97 & 0.981 & 0.949 & $\boldsymbol{C}$ \\
\hline 0.927 & 0 & 1 & 0.927 & 0.962 & 0.959 & 0.977 & 0.951 & $\boldsymbol{D}$ \\
\hline 0.982 & 0.008 & 0.931 & 0.982 & 0.956 & 0.951 & 0.99 & 0.977 & $\boldsymbol{E}$ \\
\hline 0.982 & 0.006 & 0.947 & 0.982 & 0.964 & 0.96 & 0.996 & 0.938 & $\boldsymbol{F}$ \\
\hline 0.945 & 0.01 & 0.912 & 0.945 & 0.929 & 0.921 & 0.966 & 0.836 & $\boldsymbol{G}$ \\
\hline 0.982 & 0.002 & 0.982 & 0.982 & 0.982 & 0.980 & 0.991 & 0.982 & $\boldsymbol{H}$ \\
\hline 0.945 & 0.004 & 0.963 & 0.945 & 0.954 & 0.949 & 0.988 & 0.965 & $\boldsymbol{I}$ \\
\hline 0.945 & 0.004 & 0.963 & 0945 & 0.954 & 0.949 & 0.989 & 0.953 & $\boldsymbol{J}$ \\
\hline 0.965 & 0.004 & 0.966 & 0.965 & 0.966 & 0.962 & 0.987 & 0.952 & Wt.Avg \\
\hline A: TP Rate; B: FP Rate; C: Precision; D: Recall; E: F-Measure; F: \\
\hline \multicolumn{7}{|c|}{ MCC; G: ROC Area; H: PRC Area. } \\
\hline
\end{tabular}

Table 2. Detailed accuracy by class - RIPPER algorithm

\subsection{Feature Classification using FURIA algorithm}

The contributing bin ranges from the $59^{\text {th }}$ bin were classified using the FURIA algorithm. As discussed, the default rule set is initially used to train all the data set without pruning. In this FURIA strategy, 13 rules were generated for classification using rule stretching approach.

\section{FURIA rules:}

1. (H30 in [1692, 2284, inf, inf]) and (H27 in [-inf, inf, 4,220$]) \Rightarrow$ Condition $=\mathrm{GOOD}(\mathrm{CF}=0.97)$

2. (H28 in $[1375,2075$, inf, inf $])$ and $(\mathrm{H} 27$ in [68, 220$, inf, inf $]) \Rightarrow$ Condition $=$ AIR $(\mathrm{CF}=0.97)$

3. (H30 in [-inf, -inf, 504, 522]) and (H28 in [580, 598, inf, inf]) and (H24 in [-inf, -inf, 0, 312]) $\Rightarrow$ Condition=BOS $(\mathrm{CF}=0.97)$

4. (H28 in [1181, 1203, inf, inf]) and (H29 in [5724, 6537, inf, inf] $) \Rightarrow$ Condition=DPWI $(\mathrm{CF}=0.97)$

5. $(\mathrm{H} 29$ in $[-$ inf, -inf, 1235, 4317]) $\Rightarrow$ Condition=DPWIO $(\mathrm{CF}=0.97)$
6. (H28 in [839, 935, inf, inf]) and (H29 in [1235, 7895, inf, inf]) and (H28 in [-inf, -inf, 1130, $1134]) \Rightarrow$ Condition $=$ UDPWI $(\mathrm{CF}=0.96)$

7. (H30 in [669, 713, inf, inf]) and (H28 in [-inf, -inf, $1181,1203])$ and (H28 in [1041, 1141, inf, inf]) $\Rightarrow$ Condition=UDPWI $(\mathrm{CF}=0.92)$

8. (H29 in [8366, 8458, inf, inf]) and (H28 in [222, 326, inf, inf]) and (H28 in [-inf, -inf, 580, 598]) and (H27 in [-inf, -inf, 0, 4]) $\Rightarrow$ Condition=UDPWIO $(\mathrm{CF}=0.96)$

9. (H29 in [8366, 8458, inf, inf]) and (H29 in [-inf, inf, 8846, 9013]) and (H30 in [504, 537, inf, inf]) $\Rightarrow$ Condition $=$ UDPWIO $(\mathrm{CF}=0.93)$

10. (H30 in [-inf, -inf, 80, 116]) and (H22 in [-inf, -inf, $11,63]) \Rightarrow$ Condition $=\mathrm{DRPW}(\mathrm{CF}=0.97)$

11. (H29 in $[638,9585$, inf, inf $])$ and (H30 in $[80,116$, inf, inf]) and (H30 in [-inf, -inf, 262, 267]) $\Rightarrow$ Condition=DRMF $(\mathrm{CF}=0.97)$

12. (H29 in [9490, 9523, inf, inf]) and (H28 in [109, 111 , inf, inf $]) \Rightarrow$ Condition=DRMF $(\mathrm{CF}=0.84)$

13. (H28 in [-inf, -inf, 222, 326]) and (H30 in [287, 301 , inf, inf]) and (H22 in [-inf, -inf, 0, 401]) $=>$ Condition=RL $(\mathrm{CF}=0.97)$

Number of Rules: 13

The selected seven features were classified using the FURIA. The results have been displayed in the form of confusion matrix as shown in Table 3. Referring the Table 3 , among the 550 considered data points, 543 data points have been correctly classified. Hence the classification accuracy is $98.73 \%$. The summary of the classification process has been given below.

$\begin{array}{lrr}\text { Total Number of data point } & 550 & \\ \text { Correctly classified } & 543 & 98.73 \% \\ \text { Misclassified } & 7 & 1.27 \% \\ \text { KS } & 0.9859 & \\ \text { MA error } & 0.0044 & \\ \text { RMS error } & 0.0527 & \\ \text { RA error } & 2.42 \% & \end{array}$

\begin{tabular}{|c|c|c|c|c|c|c|c|c|c|c|}
\hline Category & $\boldsymbol{A}$ & $\boldsymbol{B}$ & $\boldsymbol{C}$ & $\boldsymbol{D}$ & $\boldsymbol{E}$ & $\boldsymbol{F}$ & $\boldsymbol{G}$ & $\boldsymbol{H}$ & $\boldsymbol{I}$ & $\boldsymbol{J}$ \\
\hline $\boldsymbol{A}$ & 55 & 0 & 0 & 0 & 0 & 0 & 0 & 0 & 0 & 0 \\
\hline $\boldsymbol{B}$ & 0 & 55 & 0 & 0 & 0 & 0 & 0 & 0 & 0 & 0 \\
\hline $\boldsymbol{C}$ & 0 & 0 & 55 & 0 & 0 & 0 & 0 & 0 & 0 & 0 \\
\hline $\boldsymbol{D}$ & 0 & 0 & 0 & 55 & 0 & 0 & 0 & 0 & 0 & 0 \\
\hline $\boldsymbol{E}$ & 0 & 0 & 1 & 0 & 54 & 0 & 0 & 0 & 0 & 0 \\
\hline $\boldsymbol{F}$ & 0 & 0 & 0 & 0 & 0 & 55 & 0 & 0 & 0 & 0 \\
\hline $\boldsymbol{G}$ & 0 & 0 & 0 & 0 & 0 & 0 & 55 & 0 & 0 & 0 \\
\hline $\boldsymbol{H}$ & 0 & 0 & 0 & 0 & 0 & 0 & 0 & 55 & 0 & 0 \\
\hline $\boldsymbol{I}$ & 0 & 0 & 0 & 0 & 0 & 0 & 1 & 0 & 53 & 1 \\
\hline $\boldsymbol{J}$ & 0 & 0 & 0 & 0 & 0 & 0 & 2 & 0 & 2 & 51 \\
\hline
\end{tabular}

Table 3. Confusion matrix for FURIA

Compared to RIPPER, FURIA yields better classification accuracy. Moreover, in the confusion matrix, all the GOOD 
condition data points are correctly classified as GOOD. Hence among the GOOD condition data points there no misclassification. The summary of the FURIA classifier has been given below. The individual class efficiency has been given in the form of detailed accuracy by class. Table 4 shows the detailed accuracy by the class. The classification and misclassification details can be studied by referring to this table.

\begin{tabular}{|c|c|c|c|c|c|c|c|c|}
\hline $\boldsymbol{A}$ & $\boldsymbol{B}$ & $\boldsymbol{C}$ & $\boldsymbol{D}$ & $\boldsymbol{E}$ & $\boldsymbol{F}$ & $\boldsymbol{G}$ & $\boldsymbol{H}$ & $\boldsymbol{C l a s s}$ \\
\hline 1 & 0 & 1 & 1 & 1 & 1 & 1 & 1 & $\boldsymbol{A}$ \\
\hline 1 & 0 & 1 & 1 & 1 & 1 & 1 & 1 & $\boldsymbol{B}$ \\
\hline 1 & 0.002 & 0.982 & 1 & 0.991 & 0.99 & 1 & 1 & $\boldsymbol{C}$ \\
\hline 0.964 & 0 & 1 & 0.964 & 0.981 & 0.98 & 0.982 & 0.967 & $\boldsymbol{D}$ \\
\hline 0.982 & 0 & 1 & 0.982 & 0.991 & 0.99 & 1 & 1 & $\boldsymbol{E}$ \\
\hline 1 & 0.004 & 0.965 & 1 & 0.982 & 0.98 & 0.998 & 0.964 & $\boldsymbol{F}$ \\
\hline 1 & 0.002 & 0.982 & 1 & 0.991 & 0.99 & 0.999 & 0.981 & $\boldsymbol{G}$ \\
\hline 1 & 0 & 1 & 1 & 1 & 1 & 1 & 1 & $\boldsymbol{H}$ \\
\hline 0.964 & 0.004 & 0.964 & 0.964 & 0.964 & 0.96 & 0.980 & 0.932 & $\boldsymbol{I}$ \\
\hline 0.964 & 0.002 & 0.981 & 0964 & 0.972 & 0.97 & 0.981 & 0.948 & $\boldsymbol{J}$ \\
\hline 0.987 & 0.001 & 0.987 & 0.987 & 0.987 & 0.986 & 0.994 & 0.979 & $\boldsymbol{W t . A v g}$ \\
\hline
\end{tabular}

Table 4. Detailed accuracy by class

\section{Comparative Results}

The selected histogram features were classified using the RIPPER and the FURIA classifier model. Compared to RIPPER, FURIA performs better and yield maximum classification accuracy. The RIPPER is a conventional rule learner which forms a decision list. In this case, conventional rules are learned for each class. Finally, a default rule is added to the majority class. In this case, all the classes are not treated as a symmetric class; it will cause an unwanted bias. In addition sorting rules is also a difficult task. So the classification accuracy is less. The FURIA learns the training set using the fuzzy rules instead of conventional rules one vs rest scheme. The decision list is also replaced by the unordered rule set which deals with more uncovered example. Moreover, the fuzzy rules having soft boundaries whereas conventional rule sets having sharp boundaries. In addition, FURIA disables the pruning step. Therefore, the FURIA produced a more complex model than RIPPER. Hence the FURIA model produced better classification accuracy as $98.72 \%$. The generated results have also been compared with the J48 Decision tree learner which is a well-known benchmark learner.

The decision tree also uses an error reduction using information gain due to which the basic minimal covering rules were used for classification. As the model simple and produced better accuracy than the RIPPER as $96.55 \%$ as the classification accuracy. However, the FURIA outperforms both the algorithm and produced better results. Table 5 shows the comparative results. The comparative result shows the histogram feature with FURIA gives maximum classification accuracy compare to RIPPER and J48 decision tree. Hence, the histogram with FURIA can be considered as a suitable feature classifier for the real-time brake fault diagnosis study. In this study, a static model was used for the fault prediction study. As the results are encouraging, the suggested feature classifier model can also be used for predicting the faults in a real vehicle brake system as a future study.

\begin{tabular}{|c|c|}
\hline Name of the classifier & Classification Accuracy (\%) \\
\hline J48 Decision Tree & 97.82 \\
\hline RIPPER & 96.55 \\
\hline FURIA & 98.73 \\
\hline
\end{tabular}

Table 5. Comparative study

\section{Conclusion}

The vibration-based brake fault diagnosis study was conducted using FURIA and RIPPER. A static model which resembles the automobile brake system was fabricated and the vibration signatures were captured under the specified fault conditions using a piezoelectric accelerometer. The histogram features were extracted from the captured signals using the visual basic code. Among the 69 extracted bin ranges feature selection was carried out for the classification. The selected bins were then classified using the FURIA and RIPPER algorithm. The results of those algorithms were compared. The FURIA algorithm produced a better result (98.73\%) than the RIPPER (96.55\%) and J48 (97.82 \%) model. Hence, FURIA can be suggested for the real-time fault diagnosis study.

\section{REFERENCES}

Alamelu Manghai, T.M., Jegadeeshwaran, R., \& Sugumaran, V. (2018). Vibration based Condition Monitoring of a Brake System using Statistical Features with Logit Boost and Simple Logistic Algorithm. International Journal of Performability Engineering. Vol. 14(1), pp. 1-8.

Ana Palacios., Luciano Sanchez., Ines Couso., \& Sebastien Destercke. (2016). An extension of the FURIA classification algorithm to low quality data through fuzzy rankings \& its application to the early diagnosis of dyslexia. Neurocomputing. Vol. 176, pp. 60 - 71.

Bostrom, H. (2004) Pruning \& exclusion criteria for unordered incremental reduced error pruning. Proceedings of the Workshop on Advances in Rule Learning, ECML. Pp. $17-29$.

Chapelle., Olivier., Patrick Haffner., \& Vladimir, N. Vapnik. (1999). Support vector machines for histogrambased image classification. IEEE Transactions on Neural Networks. Vol. 10(5), pp. 1055 - 1064.

Eineborg, M., \& Bostrom, H. (2001). Classifying uncovered examples by rule stretching. International Conference 
on Inductive Logic Programming. Springer-Verlag. Pp. $41-50$.

Erdem, Ergin., \& Jing Shi. (2011). ARMA based approaches for forecasting the tuple of wind speed and direction. Applied Energy. Vol. 88(4), pp. 1405 - 1414.

Faris Elashaa., Matthew Greaves., David Mba., \& Abdulmajid Addali. (2015). Application of Acoustic Emission in Diagnostic of Bearing Faults within a Helicopter gearbox, Procedia CIRP, Vol. 38, pp. $30-$ 36.

Jafar Zarei., Mohammad Amin Tajeddini., \& Hamid Reza Karimi. (2014). Vibration analysis for bearing fault detection and classification using an intelligent filter. Mechatronics. Vol. 24, pp. 151-157.

Jegadeeshwaran, R., \& Sugumaran, V. (2013). Comparative study of decision tree classifier and best first tree classifier for fault diagnosis of automobile hydraulic brake system using statistical features. Measurement. Vol. 46 (9), pp. 3247 - 3260.

Jegadeeshwaran, R., \& Sugumaran, V. (2015). Fuzzy classifier with automatic rule generation for fault diagnosis of hydraulic brake system using statistical features. International Journal of Fuzzy Computation and Modeling. Vol. 1 (3), pp. 333 - 350.

Livani., Hanif., \& Yaman Evrenosoglu, C. (2011). A machine learning and wavelet-based fault location method for hybrid transmission lines. IEEE Transactions on Smart Grid. Vol. 5(1), pp. $51-59$.

Muralidharan, V., \& Sugumaran, V. (2012). A comparative study of Naïve Bayes classifier and Bayes net classifier for fault diagnosis of monoblock centrifugal pump using wavelet analysis. Applied Soft Computing. Vol. 12 (8), pp. 2023 - 2029.

Painuli, S., Elangovan, M., Sugumaran, V. (2014). Tool condition monitoring using K-star algorithm. Expert Systems with Applications. Vol. 41 (6), pp. 2638 - 2643.

Pan, X., Hu, X., Zhang, Y., Feng, K., Wang, S., Chen, L., \& Cai, Y. (2018). Identifying patients with atrioventricular septal defect in down syndrome populations by using self-normalizing neural networks and feature selection. Genes, 9(4), 208.

Pearson, K. (1895). Contributions to the Mathematical Theory of Evolution. II. Skew Variation in Homogeneous Material. Philosophical Transactions of the Royal Society A: Mathematical, Physical and Engineering Sciences. Vol. 186, pp. 343-414.

Ragini Sidar., Prakash Kumar Sen., \& Gopal Sahu. (2015). Review of Vibration Based Fault Diagnosis in Rolling Element Bearing and Vibration Analysis Techniques, International Journal of Scientific Research Engineering \& Technology, Vol. 4(10), pp. $998-1003$.

Ravikumar, S., Ramachandran, K.I., \& Sugumaran, V. (2011). Machine learning approach for automated visual inspection of machine components. Expert Systems with Applications. Vol. 38 (4), pp. 3260 - 3266.
Sakthivel N.R., Indira V., Binoy B. Nair, Sugumaran V. (2011) Use of histogram features for decision tree-based fault diagnosis of monoblock centrifugal pump, Int. J. ranular Computing, Rough Sets and Intelligent Systems, Vol. 2(1), pp. $23-36$.

Sakthivel, N.R., Sugumaran, V., \& Babudevasenapati, S. (2010) Vibration based fault diagnosis of monoblock centrifugal pump using decision tree. Expert Systems with Applications. Vol. 37(6), pp. 4040 - 4049.

Saravanan, N., Cholairajan, S., \& Ramachandran, K.I. (2009). Vibration-based fault diagnosis of spur bevel gear box using fuzzy technique, Expert Systems with Applications, Vol. 36, pp. 3119-3135.

Sohn Hoon., \& Charles, R. Farrar. (2011). Damage diagnosis using time series analysis of vibration signals. Smart materials and structures. Vol. 10(3), pp. 446 451.

Sugumaran, V., Muralidharan, V., \& Ramachandran, K.I. (2007). Feature selection using Decision Tree and classification through Proximal Support Vector Machine for fault diagnostics of roller bearing. Mechanical Systems and Signal Processing. Vol. 21(2), pp. 930 942.

\section{BIOGRAPHIES}

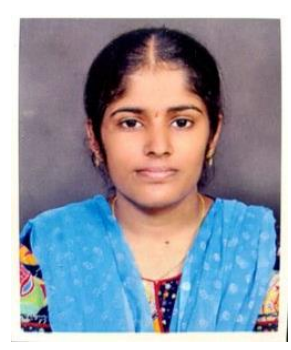

Alamelu Manghai $\mathbf{T} \mathbf{M}$ is an Junior Research fellow at School of Mechanical and Building Sciences, Vellore Institute of Technology (Deemed to be University), Chennai, India. She completed her B.E (Electrical and Electronics) and M.E (Power Electronics \& Drives) in the year 2014 and 2016. She is pursuing her doctoral degree at Vellore Institute of Technology (Deemed to be University), Chennai in the area of brake health monitoring. She has published three papers in international journals and five papers in international and national Conferences. Her research interest is condition monitoring and Fault diagnosis

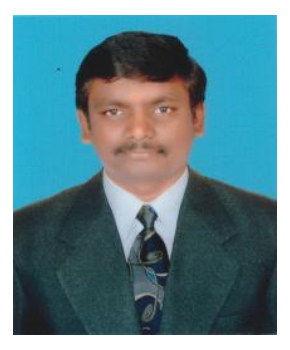

Jegadeeshwaran $\mathbf{R}$ completed his Under Graduate course in Mechanical Engineering in the year 2002. He pursued his Master's degree in Mechatronics Engineering in 2009. He completed his doctoral degree in the area of brake fault diagnosis in Vellore Institute of Technology, Chennai, Tamil Nadu. At present, he is working as an associate professor at VIT University, Chennai, Tamil $\mathrm{Nadu}$, India. He has published more than twenty five papers in peer-reviewed international Journals. He has six international / National conference publications. He is doing research in the field of fault diagnosis and condition monitoring. 